ist deshalb darauf angewiesen, durch genaue Messung von $A_{0}$ bei geeigneter Abstufung der Werte von $\mathrm{n}_{2}$ und unter Berücksichtigung der wahrscheinlichen Werte von $\delta_{1}$ auf die GröBe von $A_{1}$ zu schließen. Wären die Werte von $A_{1}$ gegeben, so ließen sich unter bestimmten $A n$ nahmen die Kurven für $A_{0}$ sowohl für Zelloidin wie für Zellulose durch Rechnung leicht feststellen und mit den durch die Beobachtung gefundenen vergleichen, was besonders für den Verlauf der Kurven, die den verschiedenen Wellenlängen zugehören, sehr wünschenswert wäre.

Es mögen hier noch einige Bemerkungen Platz finden, die zwar rein hypothetischer Art sind und mit den Untersuchungen des optischen Verhaltens yon Zalloidin und Zellulose in keinem direkten Zusammenhang stehen, die aber darauf hinweisen sollen, dab die Erweiterung der vorstehenden theoretischen Erörterungen über das Zusarnmenwirken von Stäbchen- oder Schichtendoppelbrechung mit Eigendoppelbrechung auch für eine Reihe anderer Fragen eine allgemeinere Bedeutung hat. Könnte man nämlich Systeme herștellen, bei denen die Eigendoppeibrechung beträchtlich stärker und auberdem genau bekannt wäre, - etwa sehr kleine positive oder negative stäbchenförmige Kriställchen unhülit von Flüssigkeiten - so würde man eiren klareren Ueberblick über den Einfluß der Anisotropie des festen Bestandteils anf die Gesamtdoppelbrechung erhalten. Uebrigens dürfte schon die rein theoretische Untersuchung solcher Fäle manchen interessanten Aufschlut ergeben. Noch verwickelter müBte die Sache werden, wenn beide Komponenten feste Körper mit Eigendoppelbrechung wären. Derartige Mischkörper kommen in der Natur jedenfalls häufig vor, und man darf wohl annehmen, daB bei vielen Mischkristallen und dergleichen ähnliches vorliegt und daß deshalb bei der Beurteilung der Gesamtdoppelbrechung nicht nur die Eigenschatten der Komponenten, sondern auch die notwendig hinzukommende Mitwirkung der Stäbshen- oder Schichtendoppelbrechung zu berücksichtigen wäre. Eine genauere theoretische Durcharbeitung der sich hieran anknüptenden Fragen würde wohl auch manchen Aufschlub über die Entstehung der sogen. „optischen Anomalien " geben, die ja bei solchen Mischkörpern fast stets $z u$ beobachten sind. Auch die merkwürdigen Aenderungen im optischen Verhalten, die von F. Rin ne ${ }^{8}$ ) bei dem kristallographisch-chemischen $A b$ - und Umbau besonders bei Zeolithen untersucht worden sind. gehören vielleicht zum Teil mit in dieses Gebiet. Daf bei Mischkristallen, deren optische Anomalien haufig auf "Spannungen" zurückgeführt wurden, vielmehr der periodische Aufbau der beiden Komponenten zur Erklärung jener Erscheinungen herbeigezogen werden müsse, darauf habe ich schon vor zwanzig Jahren in einer kleinen in Gemeinschaft mit $M$. Le B la n c veröffentlichten Mitteilung ${ }^{7}$ ) hingewiesen, in der ich sagte: "Sind in einem Kristalle einzelne Teilchen von verschiedener Zusammensetzung vorhanden, so wird danit auch eine von Ort zu Ort wechseind optische Dichtigkeit verbunden sein, d. h. wir werden es mit einem System zu tun haben, das aus einzelnen Schichten oder anders gestalteten Komplexen von vielleicht nicht wenig verschiedenen Brechungsexponenten besteht. In einem solchen System braucht natürlich nicht die geringste Spannung vorhanden zu sein, und doch kann sehr wohl eine optische Anisotropie sich bemerklich machen, wie sich aus rein theoretischen Grüden annehmen läBt" Das auch in organisierten Körpern von mizellarem Aufbau z. B. in vielen Membranen der Pllanzen und Tiergewebe solche Verhaltnisse vorliegen, kant wohl mit Sicherheit angerommen werden.

c) F. Rinne, Fortschr. d. Mineral., Kristallogr. u. Petrogr. 3, 159 (1913).

7) Zeitschr. 1. physik. Chem. 22, 1, $130(1897)$.

\title{
Hydrosole des Quecksilbers und seiner Sawerstoff-Verbindungen.
}

Von Con rad Amberger (Erlangen). (Eingegangen am z2. Februar 1916.) (Mitteilung aus der König!. Untersuchungs-Anstalt Erlangen.)

Von den verschiedenen organischen Schutzkolloiden ${ }^{1}$, durch deren Zusatz es gelingt, anorganische Hydrosole der verschiedenen Art zu

1) Die im Nachstehenden beschrizbenen Präparate wurden bereits vor mehreren Jahren dargestellt. Die stabilisieren, zeigen die Eiweilspaltungsprodukte - Protalbin- und Lysalbinsäure in Gestalt ihrer Alkalisalze - im allgemeinen weitaus die

Pubikation inrer Darstellungsweise muBte aus Patentrüksichten bis heute verschoben werden. 
stärkste Schutzwirkung und man kann unter Anwendung des $\mathrm{Pa}$ al'schen Verfahrens sogar anorganische Hydrosole durch Eindampfen in fester, jahrelang haltbarer, wasserlöslicher Form gewinnen, während bei Benutzung anderer Schutzkolloide, z. B. Glutin, Gummi, Dextrin zwar kolloide Lösungen erhalten werden, die aber bei dem Versuche, sie in fester Form zu erhalten, meist sofort ihren Hydrosolcharakter verlieren und in unlösliche Gele übergehen.

Besonders schwierig lassen sich dauernd haltbare, in Wasser kolloid lösliche Präparate von elementarem Quecksilber in kolloider fester Form gewinnen.

Die Darstellung von kolloidem Quecksilber ist ja schon vielfach versucht worden. Ganz verdünnte Lösungen sind zuerst von J. Billite ${ }^{2}$ ) durch elektrolytische Zerstäubung erhalten worden. A. Lottermoser ${ }^{3}$ ) hat durch Reduktion von Merkuronitrat mittelst salpetersaurem Zinnoxydul wasserlösliches, zinnsäurehaltiges Quecksilber gewonnen, das eine Zeit lang als Hyrgol pharmazeutische Verwendung fand A. Gutbie ${ }^{4}$ ) hat nur vorübergehend und unter Eintritt baldiger Sedimentation durch Reduktion sehr verdünnter Merkuronitratlösungen mit Hydraziuhydrat das stark verdünnte Hydrosol des Quecksilbers erhalten künnen. Auch mit Hilie der von $\mathrm{C}$. $\mathrm{Pa}$ al aufgefundenen Schutzkolloide der Protalbin- und Lysalbinsäure ist die Herstellung von kolloidem Quecksilber versucht worden.

Wendet man nämlich die als Schutzkolloide im allgemeinen wirksamen Natriumsalze der Protalbin- und Lysalbinsäure an, so erhält man zwar nicht durch Eindampfen, aber durch Fällung mit Alkohol die festen Adsorptionsverbindungen des kolloiden Quecksilbers mit dem betreffenden Natriumsalz. Es ist mir z. B. mit Hilfe des lysalbinsauren Natriums als Schutzkolloid wohl möglich gewesen, noch lösliche Präparate zu erhalten, die im trockenen Zustande bis zu 96 Proz. Quecksilber aufwiesen. Doch nahm die Löslichkeit dieser mit größter Sorg. falt hergestellten Präparate außerordentlich rasch $a b$ und auch die Lösungen von Präparaten mit geringerem Quecksilbergehalt sedimentierten rasch. Die im auffalienden Lichte rote Farbe der Lösung ging nach kurzer Zeit in braungrau

2) J. Billiter, Ber. d. Deutsch. chem. Ges. 35, 1929 (1902).

8) A. Lottermoser, Journ. f. prakt. Chem. [2] 57, 484 (1898).

4) A. Gutbier, Zeitschr. f. anorg. Chem. 32, 353 (1902) und 44, 228 (1905). über und die Sedimentation begann. Nur bei Anwendung von Hydrosulfiten und Alkalisulfiten als Reduktionsmittel ') resultierten Präparate von gröBerer Haltbarkeit; sie enthielten aber neben Quecksilber noch ein zweites anorganisches Kolloid, närnlich Quecksilbersulfid. Vermutlich wirkt dieses Quecksilbersulfid mit seiner hohen Beständigkeit neben den Eiweibspaltungsprodukten als zweites Schutzkolloid und wohl erst durch die gemeinsame Wirkung beider Schutzkomponenten wird die relativ hohe Beständigkeit erzielt, die den nach diesem Verfahren hergestellten Präparaten eigen war.

Es wurde beim Suchen nach therapeutisch wirksamen Quecksilberpräparaten die überraschende Beobachtung gemacht, dab unter Einhaltung bestimmter Versuchsbedingungen und unter Arwendung geeigneter Reduktionsmittel andere, im allgemeinen eine weit geringere Schutzwirkung wie Eiweib- und Eiweißspaltungsprodukte ausübende Substanzen, für die Darstellung dauernd haltbaren, kolloiden, therapeutisch wirksamen Quecksilbers vorzuiglich geeignet sind. Es können mit Gummi, Dextrin usw. kolloide Quecksilberpräparate unter bestimmten Versuchsbedingungen erhalten werden, die jahrelang haltbar sind und denen in besonders hohem Make die dem kolloiden Quecksilber charakteristischen Eigenschaften eigen sind.

Werden gewisse Albuminate wie Glutin, Gummiarten, insbesondere Dextrin oder Pflanzenschleime mit Pyrogallol, Brenzkatechin, gewissen Aminophenolen gemischt und gibt man dieser Mischung Quecksilbersalze, z. B. Quecksilberchlorid $2 u$, so entstehen weike bis gelbe Niederschläge, die durch Zusatz von Alkohol noch vermehrt werden können. Setzt man nun diesen Suspensionen Alkali zu, so erfolgt momentan Reduktion und das Quecksilber gelangt in kolloider Form zur Abscheidung und zwar ziemlich frei von Reaktionsnebenprodukten. Auffallenderweise führen nur organische Reduktionsmittel, insbesondere die mehrwertigen Phenole, zur Bildung von beständigen, kolloiden Quecksilberpräparaten, wăhrend anorganische Reduktionsmittel, wie z. B. Hydroxylamin oder Hydrazin, für diesen $Z$ weck ungeeignet sind, da durch diese schon vor der Bildung der vorerwähnten Niederschläge Reduktion zu elementarem Quecksilber erfolgt. das dann kein beständiges Hydrosol bildet.

Nach diesem Verfahren konnten Präparate in Form von festen Hydrosolen des Quecksilbers

5) Koll-Zeitschr. 8, 2, 88 (1911). 
erhalten werden mit einem Quecksilbergehalt bis zu 80 Proz. Die Präparate lösen sich leicht in Wasser zu einer im auffallenden Licht stahlblauen, in Durchsicht rotbraunen Flüssigkeit und besitzen therapeutische Wirksamkeit bei intravenöser Anwendung.

Darstellung verschiedener Quecksilberhydrosole von wechselndem Quecksilbergehalt.

1. 3,0 g reinstes, weißes Dextrin werden in $70 \mathrm{ccm}$ Wasser grelöst. Zur Lösung werden $0,4 \mathrm{~g}$ Pyrogallol in Substanz zugegeben und weiterhin eine filtrierte Lösung von1 $1,5 \mathrm{~g}$ Quecksilberchlorid in $25 \mathrm{ccm}$ Wasser. Das Ganze wird auf ein Volumen von $150 \mathrm{ccm}$ gebracht. Es entsteht zunächst cine weiße Opaleszenz, dann Trübung und Niederschlag. Gibt man nun Normalkalilauge hinzu, . so färbt sich die Mischung braun, es findet Reduktion zu elementarem Quecksilber statt, das sich bei weiterer Zugabe von Normalkalilauge (bis zu $25 \mathrm{ccm}$ ) löst zu einer im durchfallenden Licht braunroten Flüssigkeit. Uni das Kolloid in fester Form zur Abscheidung zu bringen, gibt man $200 \mathrm{ccm} 96$ prozentigen Alkohol zu und schüttelt kräftig um und läßt absitzen. Nach dem Absitzen wird die überstehende braun bis schwarzbraun gefärbte Flüssigkeit abgegossen, der Niederschlag solange mit 65 volumprozentigem Alkohol digeriert, bis er kein Alkali mehr abgibt, dann nochmal mit 96 prozentigem Alkohol gewaschen, auf einem Filter oder einer Filterplatte gesamult und an der Luft ohne Anwendung gröBerer Wärmemengen getrocknet.

Man erhält so eine feste, glänzend schwarze, körnige Masse. Das Präparat löste sich leicht in Wasser zu einer in auffallenden Lichte blauschwarzen, in durcbfallenden Lichte rotbraunen Fliissigkeit. Derartige Präparate sind in fester Form sehr lange Zcit unverändert haltbar.

Die Bestimmung des Quecksilbergehaltes in diesen Präparaten geschah in der Weise, daB eine gewisse Substanzmenge ca $0,4-0,5 \mathrm{~g}$ in ca. $50 \mathrm{ccm}$ Wasser in einem Erlenmeyer-Kolben gelöst und zur Lösung Salzsäure und chlorsaures Kali zugegeben wurden. Nach ca. 24stündigem Stehen war der gröbte Teil der organischen Substanz zerstört and das Quecksilber in das Chlorid übergeführl.

Zur völligen Beendigung des Prozesses wurde der Kolben mit einem Steigrohr verbunden und noch einige Zeit unter erneuter Zugabe von chlorsaurem Kali und Salzsäure auf dem Wasserbade erhitzt. Nach dem Erkalten wurde die überschüssige Säure mit Ammoniak abgestumpft und Schwefelwasserstoff bis zur völligen Fällung eingeleitet. Der Niederschlag wurde auf ein zuvor bei 150 " getrocknetes und tariertes quantitatives Filter. gebracht, mit AIkohol, Wasser und Schwefelkohlenstoff ausgewaschen, bei $105^{\circ}$ getrocknet und gewogen.

Angew. Subst. 0,510. Gef. $\mathrm{Hg} \mathrm{S}=0,150$

$$
=0,1293 \mathrm{Hg} \text {. Proz. } \mathrm{Hg}=25,4 \text {. }
$$

Das Präparat enthielt demnach 25,4 Proz. Quecksilber in kolloider Form.

Zur Darstellung höherprozentiger Pruparate wurde in nachstehend angegebener Weise verfahren.

Il. $1,5 \mathrm{~g}$ reines weibes Dextrin wurden in $50 \mathrm{ccm}$ Wasser gelöst. Zur Lösung wurden $0.5 \mathrm{~g}$ Pyrogallol zugegeben und weiterhin eine filtrierle Lösung von $1,5 \mathrm{~g}$ Quecksilberchlorid in ca. $20 \mathrm{ccm}$ Wasser. Das Ganze wurde gebracht auf ein Volumen von $120 \mathrm{ccm}$. Der entstehende Niederschlag wurde nun dadurch noch erheblich vermehrt, daB $155 \mathrm{ccm} 96$ prozentiger Alkohol zugefügt wurden. Gibt man nun portionsweise $25 \mathrm{ccm}$ Normalkalilauge zu, so erfolgt Reduktion zu elementarem Quecksilber, das durch die Anwesenheit des Alkohols sich im überschüssigen Alkali nicht mehr löst. Nach sehr kräftggem Umschütteln und Absitzenlassen wird die iberstehende braunrote Flüssigkeit abgegossen und der Niederschlag in der unter I. angegebenen Weise weiterbehandelt.

Analyse:

Angew. Subst. 0,2464. Gef. Hig $S=0,1276$

$$
=0,11 \mathrm{Hg} \text {. Proz. } \mathrm{Hg}=44,6 \text {. }
$$

Das Präparat enthielt demnach 44,6 Proz. $\mathrm{Hg}$ in kolloider Form. Sonst zeigte das Präparat die gleichen Eigenschaften wie sie unter I beschrieben wurden.

III. Ein noch höherprozentiges Präparat wurde dadurch erhalten, da $1,5 \mathrm{~g}$ Dextrin in $50 \mathrm{ccm}$ Wasșer gelöst wurden und hierzu $1,5 \mathrm{~g}$ Pyrogallol und eine filtrierte Lösung von $4,5 \mathrm{~g}$ Quecksilberchlorid in $50 \mathrm{ccm}$ Wasser gegeben wurde. Das Ganze wurde auf ein Volumen von $120 \mathrm{ccm}$ gebracht und der sich bildende Niederschlag durch weiteren Zusatz von $250 \mathrm{ccm}$ Alkohol vermehrt. Setzt man nunmehr in kleinen Portionen eine Lösung von $3 \mathrm{~g}$ Kaliumhydroxyd in $12 \mathrm{ccm}$ Wasser hinzu, so erfolgt Reduktion zu elementarem Quecksilber. Das ausgeschiedene Quecksilber wird weiter behandelt, wie es unter II bereits beschrieben wurde. Analyse :

Angew. Subst. 0,26 g. Gef. $\mathrm{HgS}=0,196$ $=0,169 \mathrm{Hg}$. Proz. $\mathrm{Hg}=65,1$. 
Das Präparat enthielt demnach 65,1 Proz. Quecksilber in kolloider Form.

Das Präparat löste sich noch leicht in Wasser $z u$ einer blauschwarzen Flüssigkeit, die in Durchsicht braunrot erschien. Die Löslichkeit des Präparates nahm aber in verhältnismäßig kurzer Zeit relativ rasch $a b$.

\section{Das Hydrosol des Quecksilberoxydes.}

Werden die obengenannten Schutzkolloide (Albuminoide, Pflanzenschleime, Gummiarten) ohne Zusatz eines org:nischen Reduktionsmittels, wie z. B. Pyrogaliol, mit Alkalilaugen gernischt und in diese Mischungen Quecksilberoxydsalze eingetragen, so entsteht Quecksilberoxyd als Hydrosol. Aus dieser Lösung läbt sich das kolloide Quecksilberoxyo' rein gewinnen, indem man durch Zusatz von Alkohol das Kolloid aus seiner Lösung fällt. Vor Licht geschützt ist das Präparat lange Zeit haltbar und zeigt eine therapeutische Wirksankeit bei Krankheiten, die durch Spirochäten und Gonococcen ver. anlabt sind.

Zur Darstellang dieses Hydrosols werden $3 \mathrm{~g}$ reinstes, weibes Dextrin oder aber auch eir anderes der ohen bezeichneten Schutzkolloide in ca. 50 Teilen Wasser gelöst, zur Lösung werden $0,7 \mathrm{~g}$ Aetznatron in ca. $7 \mathrm{~g}$ Wasser gelöst zugegeben und weiterhin portionsweise eine filtrierte Lösung von $1,5 \mathrm{~g}$ Quecksilberchlorid in $25 \mathrm{ccm}$ Wasser. Man erhält eine klare, gelbe Löstung, aus der durch Zusatz von 96 volumprozentigem Alkohol das Kolloid gefällt wird. Der Niederschlag wird bis zum Verschwinden der alkalischen Reaktion des Dispersionsmittels mil 65 volumprozentigem Alkohol digeriert, mit wenig absolutem Alkohol dann mit Aether nachgewaschen und bei ge wöhnlicher Temperatur ohne Anwendung gröBerer Wärmemengen getrocknet. Die ganze Darstel lungsweise hat an einem vor Tageslicht ge schützten Orte zu erfolgen, da durch die chemisch wirksamen Strahlen des Sonnenlichtes auberordentich rasch Reduktion erfolgt.

Arbeitet man an einem vor Tagesilcht geschützten Orte, so erhält man eine gelbrote körnige Masse, die sich leicht in Wasser zu einer gelbroten, klaren Flüssigkeit lost. Wird das Präparat im Dunkeln aufbewahrt, so bleibt es lange Zeit unverändert.

Die Analyse derartiger Präparate zur Bestimmung ihres Gehaltes an Quecksilber bzw Quecksilberoxyd geschah in ähnlicher Weise wie sie bei dem Quecksilberhydrosol beschrieben wurde.
Angen. Subst. 0,360 .

$$
\text { Gef. } \mathrm{HgH}=0,1438=\mathrm{Hg} 0,124
$$

$=$ Proz. $\mathrm{Hg}=34,4$ entsprechend 37 Proz. Hg O.

Das Hydrosol des Quecksilberoxyduls.

Wic feinverteiltes Quecksilberoxyd durch inniges Mischen mit Quecksilber zu Quecksilberoxydul reduziert werden kann, so kann auch eiti Hydrosol des Quecksilberoxyduls erhaiten werden, wenn das Hydiosol des Quecksilberoxydes mit dem Hydrosol des Quecksilbers in äquivalenten Mengen gemischt wird und zwar in einem derartigen Verhältnis, daß auf $216 \mathrm{HgO}$ $200 \mathrm{~g} \mathrm{Hg}$ treffer.

Im Vorstehenden wurde ein Quecksilberpräparat leschrieben mit 44,6 Proz. Hg und ein Quecksilberoxydpräparat, das 34,4 Quecksilberoxyd enthielt, Sollen beide hinsichtlich der in ilnen enthaltenen anorganischen Komponente in einem Verhältnis von $2: 2,16$ vereinigt werden, so müssen auf $1 \mathrm{~g}$ eines 44,6 Proz. Quecksilber enthaltenden Präparates 1,44 g kolloides Quecksilberoxyd in Anwendung gebracht werden, wenn das Quecksilberhydrosol 34,4 Quecksilberoxyd enthält.

In einem Porzellanmörser wurde $1 \mathrm{~g}$ eines 44,6 Proz. kolloides Quecksilber enthaltenden Präparates innig verrieben mit $1,44 \mathrm{~g}$ eines

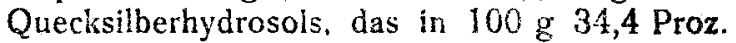
Quecksilberoxyd in kolloider form enthielt. Die gelbe Farbe der Oxydverbindung verschwindet rasch, und nach kurzer Zeit sind auch bei 500 facher Vergrößerung Teile von Quecksilberoxyd nicht mehr erkennbar, vielmehr ist unter dem Mikroskop nur eine homogene, braunschwarze Masse erkennbar. Die Vereinigung von kolloidem elemetitarein Quecksilber mit seiner Oxydverbindung zu Quecksilberoxydul war somit auch auf trockenem Wege möglich.

$\mathrm{DaB}$ eine neue Verbindung entstanden war, war auch an der Farbe der wässerigen Lösung erkennbar. Das Präparat löste sich in Wasser, wenn auch weniger leicht als ursprünglich die Einzelkomponenten, zu einer braunschwarzen Flüssigkeit, die auch in Durchsicht schwarzbraun. erschien. Läbt man eine derartige Lösung auch nur verhältnismäßig kurze Zeit dem Lichte ausgesetzt stehen, so ändert die Lösung ihre Farbe, erscheint allmählich im auffallenden Lichte blauschwarz, in Durchsicht rotbraun; schlieBlich erfolgt nach längerem Stehen Sedimentation von Quecksilber und die überstehende Flüssigkeit wird gelbrot. Dieses Kolloid ist demnach 
insbesondere in wässeriger Lösung wenig beständig und bestrebt, unter teilweiser Gelbildung in Quecksilber und Quecksilberoxyd sich umzuwandeln.
Analyse: Angew. Subst. 0,391. Gef. $\mathrm{Hg}=0.1431$

Proz. $\mathrm{Hg}=37,4=38,8 \mathrm{Hg}_{2} \mathrm{O}$.

Das Präparat enthielt demnach 38,8 Quecksilberoxydul.

\section{Chemische Reaktionen in anisotropen Flüssigkeiten.}

\section{(Zweite Mittellung.)}

Von The Svedberg (Upsala).

(Eingegangen am 20. Marz 1916.)

In der ersten gleichbetitelten Mitteilung habe ich gezeigt, $\mathrm{daB}$ eine in einer kristallinischen Flüssigkeit verlaufende chemische Reaktion bein Ueberschreiten des Klärpunktes im Sinne steigender Temperatur sprungweise ihre Geschwindigkeit vergröbert. Wenn wir also die Reaktionsgeschwindigkeit in zwei sehr nahe aneinander gelegenen Temperaturpunkten, der eine oberhalb des Klärpunktes, d. h. im isctropen Gebiete, der andere unterhalb desselben, d. h. im anisotropen Gebiete in Betracht ziehen, so ist die Reaktionsgeschwindigkeit im anisotropen Gebiete kleiner als im iso. tropen.

Im folgenden werde ich einige, Versuchsreihen mitteilen, die kaum anders gedeutet werden können als durch die Annahme, daß die Geschwindigkeit gewisser chemischer Reaktionen in einem flüssigen Kristall kleiner ist als in der entsprechendenkristallinischen Flüssig. $k$ eit ${ }^{l}$.

Als MaB der Reaktionsgeschwindigkeit diente auch in diesem Falle die zeitliche Zunahme der elektrischen Leitfähigkeit des studierten Systems. Die Messungen wurden mit der in der ersten Mitteilung erwähnten Zelle unter. Verwendung des rotierenden Kommutators ausgeführt. Mit Hilfe eines Magnetfeldes konnte die kristallinische Flüssigkeit in einen flüssigen Kristall verwandelt werden. Bei einigen Versuchen wurde zu diesem Zwecke das früher erwähnte Magnetthermostat ${ }^{2}$ ) benutzt. $\mathrm{Da}$ es aber für die in Frage stehende Untersuchung von großer Bedeutung ist, daB die Temperatur möglichst konstant gehalten werden kann, so baute juh mir

1) Ein Versuch, den ich fruher (siehe Ann. d. Phys. 1916) anstellte, um eine eventuelle Verschicdenheit zwischen einer kristallinischen Fitssigkeit und einem flussigen Kristall in bezug aul die chemische Reaktionsgeschwindigkeit aufzutinden, gab infolge der damals zu geringen Genawigkeit der Messungen ein negatives Resultat.

2) Unten als Thermostat I bezeichnet. ein neues Magnetthermostat ${ }^{3}$ ) von folgender Form (Fig. 1).

Als Thermostatgefäß diente ein versilberter Vakuumbecher von etwa 0,5 Liter Inhalt, der mit "Mobiloil B" beschickt war. Darin tauchen die Versuchszelle $Z$, ein Thermometer $T$, ein elektrisches Heizband $B$, ein Regulator $R$ und ein Glasrohr G, das zum Einblasen von Luft diente. Die Heizung wurde automatisch reguliert, so $\mathrm{daB}$ die Temperatur auf $\pm 0,05^{\circ}$ konstant gehalten werden konnte. Das Einblasen von Luft diente zum Rühren des Oels und geschah durch ein kleines Wasserstrahlgebläse. Der Vakuumbecher ist mit einem bleiernen Kühlschlauch $K$ in 30 Windungen bewickelt und ruht innerhalb der Spule $S$ auf der Asbestplatte $A$ und dem Glasfuß F. Die Spule enthält pro Zentimeter 50 Windungen eines $2 \mathrm{~mm}$ dicken blanken Kupferdrahts auf 10 Schichten verteilt, die yoneinander durch Asbestpappe isoliert sind. Die einzelnen Windungen sind nicht voneinander isoliert. Bei einer derartigen Bewickelung geht natürlich ein Teil des durch die Spule gesandten Stromes direkt zwischen den Windungen über und ist für die Erzeugung des magnetischen Feldes verloren ${ }^{4}$ ), anderseits wird es aber eben infolge des Weglassens der Isolation möglich, die Spule sehr stark -- bis zu 30 Amp. - zu belasten, ohne ein Verbrennen zu befürchten ${ }^{5}$ ).

Ein sehr konstantes und immer gut reproduzierbares Feld ist kaum in dieser Weise zu erreíchen, für einige orientierende Versuche, wie die unten mitgeteilten, ist aber eine solche Spule der leichten Herstellung und des hohen magnetischen Effektes wegen recht empfehlenswert.

i) Unten als Thermostat II bezeichnet.

4) Wenn der Dralt, wie in diesem Falle, in einer geraden Zahl von Scinchten aufgewickelt ist, so stören diese vagabundierenden Ströme wicht merklich das axiale Magnetfeld innerhalb der Spule.

5) Die Spule wurde von dem hiesigen Universitätsmechaniker, Akt.-Ges., J. L. Rose hergestellt. 